\title{
Usefulness of Exercise for Home Blood Pressure Control in People with Diabetes: A Study Protocol for a Crossover Randomized Controlled
} Trial

\author{
Keiko Iwai (iD) \\ Emi Ushigome \\ Isao Yokota (iD ${ }^{2}$ \\ Saori Majima' \\ Naoko Nakanishi ${ }^{1}$ \\ Yoshitaka Hashimoto (D) \\ Hiroshi Okada $\mathbb{1}^{3}$ \\ Takafumi Senmaru' \\ Masahide Hamaguchi ${ }^{1}$ \\ Mai Asano' \\ Masahiro Yamazaki' \\ Michiaki Fukui' \\ 'Department of Endocrinology and \\ Metabolism, Graduate School of Medical \\ Science, Kyoto Prefectural University of \\ Medicine, Kamigyo-ku, Kyoto 602-8566, \\ Japan; ${ }^{2}$ Department of Biostatistics, \\ Graduate School of Medicine, Hokkaido \\ University, Sapporo, Hokkaido 060-0808, \\ Japan; ${ }^{3}$ Department of Endocrinology and \\ Diabetology, Matsushita Memorial \\ Hospital, Moriguchi, Osaka, 570-8540, \\ Japan
}

This article was published in the following Dove Press journal:

Diabetes, Metabolic Syndrome and Obesity: Targets and Therapy

Background: The coexistence of hypertension and diabetes mellitus significantly increases the risk of macrovascular complications in patients with diabetes. Home blood pressure is important because it is more strongly associated with target organ damage and total mortality than clinic blood pressure measurements. Regular moderate aerobic exercise has antihypertensive effects. This study aims to examine the effect of aerobic exercise therapy on blood pressure at home in patients with diabetes.

Patients and Methods: In this crossover randomized controlled trial, 110 patients with type 2 diabetes will be randomly assigned to two groups: an exercise preceding group and an exercise lagging group. During the exercise period, patients will be instructed to walk either 30 minutes twice each day or 60 minutes once each day for at least 3 days per week. During the non-exercise period, patients will be permitted to perform physical activity associated with activities of daily life. Patients will be followed up for 56 days. The primary outcome will be the mean nocturnal blood pressure from day 24 to 28 during the exercise period compared to that during the non-exercise period. The secondary outcome will include variation in nocturnal blood pressure, mean blood pressure values and variation of morning and evening blood pressure from day 24 to 28 in the exercise period compared to the nonexercise period, body weight, body mass index and bioelectrical impedance analysis. Biochemical tests such as hemoglobin Alc level, blood glucose level, serum lipid profile, renal function, uric acid levels and liver function tests will also be measured.

Discussion: This randomized controlled trial will provide critical information regarding the effectiveness of moderate exercise on nocturnal blood pressure reduction. If successful, the results of this randomized controlled trial may increase exercise motivation in people with diabetes and may lead to improvement or prevention of target organ damage and overall mortality.

Trial Registration: Trial registration: University Hospital Medical Information Network, UMIN 000035973. Protocol version number: R000040969. Registration date: February 22, 2019. Recruitment began: June 19, 2019. The date of completion of recruitment: July 3, 2020 URL: http://www.umin.ac.jp.

Keywords: nocturnal blood pressure, type 2 diabetes, aerobic exercise, randomized controlled trial

Emi Ushigome Department of Endocrinology and Metabolism, Graduate School of Medical Science, Kyoto Prefectural University of Medicine, 465, Kajii Cho, Kamigyo-ku, Kyoto-city, Kyoto 62 I-8585, Japan

Tel $+8|-75-25|-5505$

Fax +8I-75-252-372I

Email emis@koto.kpu-m.ac.jp

\section{Introduction}

Hypertension is reported in more than half of the patients with diabetes mellitus, and it significantly increases the risk of both microvascular and macrovascular 
diseases. ${ }^{1}$ In particular, the coexistence of hypertension and diabetes significantly increases the risk of macrovascular diseases such as cerebral and myocardial infarctions. $^{2,3}$ Therefore, intensive monitoring and treatment for hypertension and hyperglycemia is important for patients with diabetes. Home blood pressure (HBP) is very important because it is more strongly associated with target organ damage, cardiovascular events, and total mortality than clinic blood pressure (BP). ${ }^{4}$ It is also more reliable, since individuals spend more time at home than in clinics.

Bertani et al reported that in elderly hypertensive patients, a greater nocturnal decline in diastolic BP was observed in resistance training in comparison with interval aerobic training. ${ }^{5}$ However, this study differs from their study in that they excluded patients with diabetes and with systolic $\mathrm{BP} \geq 160 \mathrm{mmHg}$ and diastolic $\mathrm{BP} \geq 100 \mathrm{mmHg}$. Aerobic exercise is currently recommended for decreasing cardiovascular risk by decreasing resting BP. An acute bout of moderate aerobic exercise elicits a decrease in BP known as post-exercise hypotension. ${ }^{6}$ This lowering of BPs is observed for up to 24 hours post-exercise, demonstrating the clinical effects of aerobic exercise in hypertensive patients. ${ }^{7}$ However, there have been no studies to investigate the effects of exercise therapy on nocturnal BP in patients with diabetes. The aim of this study is to examine the usefulness of exercise therapy in controlling HBP, especially nocturnal BP, in diabetes patients.

\section{Patients and Methods Study Design}

This study will be a randomized crossover trial aimed at evaluating the usefulness of exercise therapy in controlling HBP. It will examine the superiority of randomized comparisons between two groups of diabetes patients: an exercise preceding group (in which the exercise period is from day 1 to day 28) and exercise lagging group (exercise period is from day 29 to day 56). ${ }^{8,17}$ A total of 110 patients with type 2 diabetes who receive outpatient treatment at the Hospital of the Kyoto Prefectural University of Medicine, Japan, will be randomly assigned to the two groups. Blood samples, spot urine tests, physical measurements, and body composition, analyzed by InBody 720 bioelectrical impedance device (InBody Japan, Tokyo, Japan), will be evaluated at the baseline visit and each follow-up visit (day 29 and day 57 after enrolment). The primary outcome will be the average value of nocturnal BP from day 24 to 28 during the exercise period compared to the non-exercise period. Each participant will be enrolled for 2 months from randomization to the final follow-up assessment.

\section{Study Setting}

The study will be conducted at the Hospital of the Kyoto Prefectural University of Medicine. Recruitment by clinicians and all study visits will be conducted in the outpatient clinics.

\section{Recruitment}

Participants will be recruited from June 2019 to March 2021. First, the outpatient clinician will recruit the patient. The researcher will explain the details of the study in a separate room using the study protocol (Supplementary material).

\section{Inclusion Criteria}

The target patients must meet all of the following inclusion criteria when giving informed consent:

- Diagnosed with type 2 diabetes mellitus (we will apply the Japan Diabetes Society's 'Report of the Committee on the Classification and Diagnostic Criteria of Diabetes Mellitus' for diagnosing diabetes) ${ }^{9}$

- Aged between 20 and 90 years.

- Written informed consent must be obtained from the patient before registration in this study.

The patients were kept on the same treatment for at least 3 months prior to randomization to account for the effect of SGLT2 and GLP1 on BP. ${ }^{10,11}$

\section{Exclusion Criteria}

Patients will be excluded from the study if they fulfil any of the following exclusion criteria:

- Urine ketones moderately positive.

- Presence of advanced renal failure (serum creatinine $\geq 2.0 \mathrm{mg} / \mathrm{dL}$ or patient receiving dialysis).

- Serious diabetic autonomic neuropathy (eg cardiac autonomic neuropathy, increasing the risk of sudden cardiac arrest) or recent fundus hemorrhage due to proliferative retinopathy.

- Serious liver disease (eg decompensated cases with jaundice and ascites) or endocrine disease. 
- Serious heart disease (eg unstable angina pectoris, myocarditis, moderate to critical aortic stenosis, severe arrhythmia), lung disease, cerebrovascular disease, or orthopedic disease making exercise difficult to perform.

- Participation in the study is considered by their physician to be inappropriate for other safety reasons.

\section{Study Procedures}

After confirming that a patient is eligible for the study, written informed consent will be obtained. All participants will be asked to attend follow-up visits 1 and 2 months after enrollment.

At enrollment, the patients will be randomized to either the exercise preceding group or the exercise lagging group. Patients assigned to the exercise preceding group will be instructed to walk 30 minutes twice each day or 60 minutes once each day for at least 3 days per week from day 1 to day 28. After the follow-up visits 1 month after enrolment, patients will be instructed to perform physical activity only up to the level of daily life $(<4$ metabolic equivalents of task [METs] $)^{12}$ from day 29 to day 56 until the follow-up visits 2 months after enrollment. Patients assigned to the exercise lagging group will be instructed to perform physical activity only up to the level of daily life ( $<4$ METs) from day 1 to day 28. After the follow-up visits 1 month after enrollment, patients will be instructed to exercise at least 3 days per week from day 29 to day 56 until the follow-up visits 2 months after enrollment.

At each follow-up visit, the researcher will meet with the participants, check the exercise compliance, assess changes in physical condition, and answer participants' questions regarding the study to promote participant retention and complete follow-up. Participants' compliance during the exercise and non-exercise periods will be confirmed using questionnaire records. The questionnaire records will be collected at the second follow-up visit at the end of the study.

\section{Sample Size}

Carlson et al reported in a previous interventional study of hypertensive patients that exercise intervention (ie 4 sets of 2-minute isometric handgrip exercises separated by 3-minute rest periods, 3 days per week for 8 weeks) reduced the systolic BP by approximately $7 \mathrm{mmHg}$, from $136 \pm 12 \mathrm{mmHg}$ to $129 \pm 15 \mathrm{mmHg}(\mathrm{p}=0.04) .{ }^{13}$ Based on this report, we set the standard deviation of systolic BP in each arm to $15 \mathrm{mmHg}$ and the difference between arms to $6 \mathrm{mmHg}$. To be conservative, the correlation of arms was set to 0 . With the one-sided significance level set at 0.025 , and power set at 0.8 , the required sample size was 101 participants as determined in the paired $t$-test performed by SAS $^{\circledR}$ 9.4. Assuming a $10 \%$ dropout rate, we set the total sample size as 110 participants.

\section{Randomization Procedures}

Immediately after obtaining participants' informed consent, a third party which is not involved in this research will perform simple randomization using random numbers. All participants will be randomized to one of the two arms (exercise preceding group or exercise lagging group) in equal numbers. If the participant requests to switch the exercise period and the non-exercise period for their own convenience, unblinding is permissible, and the participant's allocated intervention during the trial will be revealed to explain that the order cannot be switched.

\section{Intervention}

Instructions on exercise intensity during the exercise period and activities of daily living permitted during the nonexercise period will be based on the METs table. ${ }^{14}$

\section{Details of Exercise Period}

The exercise therapy will consist of walking 30 minutes twice per day, or walking 60 minutes once per day, with a goal of over 6000 steps per day, for more than 3 days per week.

\section{Details of Non-Exercise Period}

During the non-exercise period, physical activity up to the level of daily life $(<4 \text { METs })^{15}$ such as stretching at home, slow walking on flat ground, commuting by bicycle, and housework will be permitted.

\section{Details of BP Measurement}

A validated, automatic device (HEM-7080IC; Omron Healthcare Co. Ltd, Kyoto, Japan) equipped with memory to store the date, time, and readings will be loaned to each participant at baseline (first outpatient visit). At this time, the researcher will also instruct participants on how to measure BP.

\section{HBP Measurements}

HBP will be measured from day 24 to day 28 of each period (exercise and non-exercise). Patients will be 
instructed to perform triplicate morning and evening BP measurements themselves, with at least 1 minute between recordings, for 5 consecutive days. Morning BP measurements will be performed within 1 hour of waking, before breakfast or taking any medicines, with the patient seated and having rested for at least 5 minutes. ${ }^{16}$ Evening BP measurements will be obtained in a similar manner just prior to bedtime. The cuff will be placed directly around the non-dominant arm, and the position of the cuff will be maintained at the level of the heart.

\section{Nocturnal BP}

Patients will be instructed to go to bed with the cuff attached to the non-dominant upper arm, and the machine will automatically measure BP at night: at 2:00, 3:00, 4:00, and 5:00 AM.

\section{Measurements and Outcomes}

All outcome measures will be assessed at baseline and at the 1-month and 2-month follow-up visits (Table 1).

\section{Primary Outcome}

The primary outcome measure will be the average value of nocturnal BP from day 24 to 28 during the exercise period compared to the non-exercise period.

\section{Secondary Outcomes}

The secondary outcomes measures will include the variation in nocturnal BP, mean blood pressure value and variation of morning and evening BP from day 24 to day 28 of the exercise period compared to the non-exercise period, hemoglobin A1c level, blood glucose level, serum lipid profile (triglycerides, total cholesterol, high-density lipoprotein cholesterol, and low-density lipoprotein cholesterol), renal function (creatinine and estimated glomerular filtration rate), liver function (alanine aminotransferase, $\gamma$-glutamyl transpeptidase, and aspartate aminotransferase), urinary albumin excretion level, sleep time, body weight, body mass index, and bioelectrical impedance analysis measured by InBody $720 .{ }^{17}$ The average sleep time will be confirmed using a questionnaire at the time of study entry.

Blood samples for biochemical measurements will be taken in the morning. Hemoglobin Alc level, serum lipid profile, and other biochemical data will be determined using standard laboratory measurements. Hemoglobin A1c level will be expressed as National Glycohemoglobin Standardization Program units. Information on sex, age, duration of diabetes, smoking status, alcohol consumption, and use of antihypertensive medication and complications of diabetes will be collected from each participant at baseline.

Neuropathy will be defined based on the diagnostic criteria for diabetic polyneuropathy proposed by the Diabetic Neuropathy Study Group. ${ }^{18}$ The presence of retinopathy will be assessed from chart reviews. Macrovascular complications will be defined as the presence of past cerebrovascular disease, cardiovascular disease, or arteriosclerosis obliterans, based on physical examination or medical history. Patients who have not undergone electrocardiography and pulse wave velocity measurement 6 months prior to baseline will undergo these before enrollment to ensure that there is no major vascular disease that would make exercise difficult. Major vascular diseases that would make it difficult to exercise include obstructive arteriosclerosis and severe arrhythmias.

Change in diabetic medication (number of drugs currently prescribed to the patient dosage of drugs initiation of new medication during study period initiation of injectable diabetic medication initiation of insulin number of medicines stopped or changed during the study period) and change in antihypertensive medication (number of drugs started or stopped during study period dosage of drugs initiation of new medication during study period) will be recorded.

\section{Statistical Methods}

A paired $t$-test will be used to test the null hypothesis that the exercise lagging group and exercise preceding group have equivalent primary endpoints. Subgroup analyses will be conducted, stratified by change in nocturnal BP by sleep time ( $<6$ or $\geq 6$ hours), ${ }^{19}$ age ( $<65$ or $\geq 65$ years), sex (male or female), and hemoglobin A1c levels $(<7 \%$ or $\geq 7 \%$ ). JMP version 14.2.0 (SAS Institute Inc., Cary, NC, USA) will be used for statistical analyses, and $\mathrm{p}<0.025$ (one-sided) will be considered statistically significant.

\section{Discussion}

The coexistence of high BP and diabetes significantly increases the risk of macrovascular disease such as cerebral infarction and myocardial infarctions., ${ }^{2,3}$

Moreover, a number of intervention studies have reported the improvement of BP by exercise therapy. ${ }^{20,21}$ This study is a randomized controlled trial on the usefulness of exercise therapy in controlling nocturnal BP in patients with diabetes. Demonstrating that moderate exercise therapy is effective to nocturnal BP in diabetic patients would have great clinical significance, since the coexistence of hypertension and 
Table I Overview of Assessment Schedule and Measures

\begin{tabular}{|c|c|c|c|c|}
\hline \multirow[b]{2}{*}{ Timepoint } & \multirow[b]{2}{*}{ Enrolment } & \multirow[b]{2}{*}{ Allocation } & \multicolumn{2}{|c|}{ Study Follow-Up Visits } \\
\hline & & & I Month & 2 Months \\
\hline $\begin{array}{l}\text { Enrolment } \\
\text { Eligibility screen } \\
\text { Informed consent } \\
\text { Allocation }\end{array}$ & $\begin{array}{l}X \\
x\end{array}$ & $\mathrm{x}$ & & \\
\hline $\begin{array}{l}\text { Exercise preceding group } \\
\text { Exercise period }\end{array}$ & & & $x$ & \\
\hline $\begin{array}{l}\text { Exercise lagging group } \\
\text { Exercise period }\end{array}$ & & & & $x$ \\
\hline $\begin{array}{l}\text { HBP measurement } \\
\text { (day 24-28 each period) }\end{array}$ & & & $x$ & $x$ \\
\hline Assessments & & & & \\
\hline Demographics & $x$ & & & \\
\hline InBody & $x$ & & $\mathrm{X}$ & $x$ \\
\hline Sleeping habits & $x$ & & $x$ & $x$ \\
\hline Medication review & $x$ & & $\mathrm{X}$ & $\mathrm{X}$ \\
\hline Body weight & $x$ & & $\mathrm{X}$ & $\mathrm{X}$ \\
\hline Hemoglobin Alc & X & & $\mathrm{X}$ & $\mathrm{x}$ \\
\hline Blood glucose & $x$ & & $x$ & $x$ \\
\hline Serum lipid profile & $x$ & & $x$ & $x$ \\
\hline Renal function & $x$ & & $x$ & $x$ \\
\hline Liver function & $x$ & & $x$ & $x$ \\
\hline Urinary albumin excretion & $x$ & & $x$ & $\mathrm{x}$ \\
\hline
\end{tabular}

Abbreviation: HBP, home blood pressure.

diabetes mellitus significantly increases the risk of macrovascular complications of diabetes. This study aims to conduct a clinical trial that will provide clinicians with evidence on how moderate exercise can affect nocturnal blood pressure. If our exercise intervention proves successful, the results of this randomized controlled trial may increase exercise motivation in people with diabetes, and may lead to improvement of target organ damage and overall mortality. This protocol may also provide guidance to other researchers interested in testing similar interventions.

The results of our study will be presented at national and international conferences and published in academic journals.

\section{Abbreviations}

HBP, home blood pressure; BP, blood pressure; METs, metabolic equivalents of task.

\section{Data Sharing Statement}

- Whether individual deidentified participant data (including data dictionaries) will be shared; No.
- What data in particular will be shared; Not available.

- Whether additional, related documents will be available; Not available.

- When the data will become available and for how long; Not applicable.

- By what access criteria data will be shared (including with whom, for what types of analyses, and by what mechanism); Not applicable.

\section{Ethics Approval and Informed Consent}

This study will be conducted in accordance with the Declaration of Helsinki. After screening for eligibility, written informed consent will be obtained from each participant by the researcher. Protocol modifications will be approved by the medical research ethics committee of the Hospital of the Kyoto Prefectural University of Medicine (protocol reference number: R000040969). This study has been registered in the University Hospital Medical Information Network clinical trials registry (UMIN000 035973). The results of this research will be announced 
at paper presentations and at academic conferences in Japan and overseas.

\section{Acknowledgments}

We would like to thank Editage (www.editage.com) for English language editing.

\section{Author Contributions}

EU, SM, NN, YH, TS, MH, MA, MY and MF are the main study physician in Graduate School of Medical Science, Kyoto Prefectural University of Medicine. MF is the principal investigator of the Graduate School of Medical Science, Kyoto Prefectural University of Medicine. KI is the guarantor of this work and, as such has full access to all the data in the study and will take responsibility for the integrity of the data and the accuracy of the data analysis. Each author listed on an article meets all of the following criteria:

- Made a significant contribution to the work reported, whether that is in the conception, study design, execution, acquisition of data, analysis and interpretation, or in all these areas.

- Have drafted or written, or substantially revised or critically reviewed the article.

- Have agreed on the journal to which the article will be submitted.

- Reviewed and agreed on all versions of the article before submission, during revision, the final version accepted for publication, and any significant changes introduced at the proofing stage.

- Agree to take responsibility and be accountable for the contents of the article.

\section{Funding}

This research received no specific grant from any funding agency in the public, commercial or not-for-profit sectors. This trial was conducted with no external funding and was instead funded from operating support funds.

\section{Disclosure}

Emi Ushigome reports personal fees from Nippon Boehringer Ingelheim Co., Ltd., personal fees from Mitsubishi Tanabe Pharma Corporation, personal fees from DAIICHI SANKYO COMPANY, LIMITED, personal fees from Takeda Pharmaceutical Company Limited, personal fees from Astellas Pharma Inc., personal fees from MSD K.K., personal fees from Kyowa Hakko Kirin
Co., Ltd., personal fees from Sumitomo Dainippon Pharma Co., Ltd., personal fees from KOWA PHARMACEUTICAL COMPANY LTD., personal fees from Novo Nordisk Pharma Ltd., personal fees from Taisho Pharmaceutical Co., Ltd., personal fees from AstraZeneca K.K., grants from ONO PHARMACEUTICAL CO., LTD., outside the submitted work.

Isao Yokota reports personal fees from Chugai Phamaceuticals, personal fees from Japan Tabacco Inc. Pharamaceutical Division, personal fees from Nippon Shinyaku, outside the submitted work.

Yoshitaka Hashimoto reports grants from Asahi Kasei Pharma and personal fees from Mitsubishi Tanabe Pharma Corp., Novo Nordisk Pharma Ltd., Sanofi K.K., and Daiichi Sankyo Co. Ltd., outside the submitted work.

Takafumi Senmaru reports personal fees from Ono Pharma Co., Ltd, personal fees from Mitsubishi Tanabe Pharma Co, personal fees from Astellas Pharma Inc, personal fees from Kyowa Hakko Kirin Co., Ltd, personal fees from Sanofi K. K., personal fees from MSD K.K., personal fees from Kowa Pharma Co., Ltd., personal fees from Taisho Toyama Pharma Co., Ltd., personal fees from Takeda Pharma Co., Ltd., personal fees from Kissei Pharma Co., Ltd., personal fees from Novo Nordisk Pharma Ltd., personal fees from Eli Lilly Japan K.K., outside the submitted work.

Masahide Hamaguchi reports grants from Asahi Kasei Pharma, grants from Nippon Boehringer Ingelheim Co., Ltd., grants from Mitsubishi Tanabe Pharma Corporation, grants from Daiichi Sankyo Co., Ltd., grants from Sanofi K.K., grants from Takeda Pharmaceutical Company Limited, grants from Astellas Pharma Inc., grants from Kyowa Kirin Co., Ltd., grants from Sumitomo Dainippon Pharma Co., Ltd., grants from Novo Nordisk Pharma Ltd., grants from Eli Lilly Japan K.K., outside the submitted work.

Masahiro Yamazaki reports personal fees from MSD K. K., personal fees from Sumitomo Dainippon Pharma Co., Ltd., personal fees from Kowa Company, personal fees from AstraZeneca PLC, personal fees from Takeda Pharmaceutical Company, personal fees from Kyowa Hakko Kirin Co., Ltd., personal fees from Daiichi Sankyo Co., Ltd., personal fees from Ono Pharmaceutical Co., Ltd., personal fees from Takeda Pharmaceutical Co., Ltd., outside the submitted work. The authors report no other conflicts of interest in this work. 


\section{References}

1. Sowers JR. Diabetes mellitus and vascular disease. Hypertension. 2013;61:943-947.

2. Kengne AP, Patel A, Barzi F, et al. Systolic blood pressure, diabetes and the risk of cardiovascular diseases in the Asia-Pacific region. J Hypertens. 2007;25(6):1205-1213.

3. Research Group N, NIPPON DATA80 Research Group. Risk assessment chart for death from cardiovascular disease based on a 19-year follow-up study of a Japanese representative population. Circ J. 2006;70(10):1249-1255. doi:10.1253/circj.70.1249

4. Niiranen TJ, Hänninen MR, Johansson J, Reunanen A, Jula AM. Home measured blood pressure is a stronger predictor of cardiovascular risk than office blood pressure: the Finn-Home study. Hypertension. 2010;55(6):1346-1351. doi:10.1161/HYPERTEN SIONAHA.109.149336

5. Bertani RF, Campos GO, Perseguin DM, et al. Resistance exercise training is more effective than interval aerobic training in reducing blood pressure during sleep in hypertensive elderly patients. $J$ Strength Cond Res. 2018;32(7):2085-2090. doi:10.1519/ JSC.0000000000002354

6. MacDonald JR, MacDougall JD, Hogben CD. The effects of exercise duration on post-exercise hypotension. J Hum Hypertens. 2000;14 (2):125-129. doi:10.1038/sj.jhh.1000953

7. Pescatello LS, Franklin BA, Fagard R, Farquhar WB, Kelley GA, Ray CA. American College of Sports Medicine position stand. exercise and hypertension. Med Sci Sports Exerc. 2004;36(3):533-553. doi:10.1249/01.MSS.0000115224.88514.3A

8. American Diabetes A. Lifestyle management: standards of medical care in diabetes-2018. Diabetes Care. 41:S38-S50.

9. Seino Y, Nanjo K, Tajima N, et al. Report of the committee on the classification and diagnostic criteria of diabetes mellitus. $J$ Diabetes Investig. 2010;1:212-228. doi:10.1111/j.2040-1124.2010.00074.x

10. Scheen AJ, Delanaye P. Effects of reducing blood pressure on renal outcomes in patients with type 2 diabetes: focus on SGLT2 inhibitors and EMPA-REG OUTCOME. Diabetes Metab. 2017;43(2):99-109. doi:10.1016/j.diabet.2016.12.010

11. Boyle JG, Livingstone R, Petrie JR. Cardiovascular benefits of GLP-1 agonists in type 2 diabetes: a comparative review. Clin Sci. 2018;132(15):1699-1709. doi:10.1042/CS20171299
12. Cardoso CR, Maia MD, de Oliveira FP, Leite NC, Salles GF. High fitness is associated with a better cardiovascular risk profile in patients with type 2 diabetes mellitus. Hypertens Res. 2011;34 (7):856-861. doi:10.1038/hr.2011.50

13. Carlson DJ, Inder J, Palanisamy SK, McFarlane JR, Dieberg G, Smart NA. The efficacy of isometric resistance training utilizing handgrip exercise for blood pressure management. A randomized trial. Medicine. 2016;95(52):e5791. doi:10.1097/MD.00000000000 05791

14. Ainsworth BE, Haskell WL, Whitt MC, et al. Compendium of physical activities: an update of activity codes and MET intensities. Med Sci Sports Exerc. 2000;32(9 Suppl):S498-504. doi:10.1097/ 00005768-200009001-00009

15. Zheng J, Cheng J, Zheng S, et al. Physical exercise and its protective effects on diabetic cardiomyopathy: what is the evidence? Front Endocrinol. 2018;3(9):729. doi:10.3389/fendo.2018.00729

16. Imai Y, Otsuka K, Kawano Y, et al. Japanese Society of Hypertension. Japanese Society of Hypertension (JSH) guidelines for self-monitoring of blood pressure at home. Hypertens Res. 2003;26:771-782. doi:10.1291/hypres.26.771

17. Hashimoto Y, Osaka T, Fukuda T, Tanaka M, Yamazaki M, Fukui M. The relationship between hepatic steatosis and skeletal muscle mass index in men with type 2 diabetes. Endocr J. 2016;63:877-884. doi:10.1507/endocrj.EJ16-0124

18. Shin SK, Pack SP, Oh JG, et al. Anti-erythropoietin and anti-thrombopoietin antibodies induced after administration of recombinant human erythropoietin. Int Immunopharmacol. 2011;11:2237-2241. doi:10.1016/j.intimp.2011.10.014

19. Kobayashi D, Kuriyama N, Osugi Y, Arioka H, Takahashi O. Longitudinal relationships between cardiovascular events, risk factors, and time-dependent sleep duration. Cardiol J. 2018;25 (2):229-235.

20. Kobayashi R, Kasahara Y, Ikeo T, et al. Effects of different intensities and durations of aerobic exercise training on arterial stiffness. $J$ Phys Ther Sci. 2020;32(2):104-109. doi:10.1589/jpts.32.104

21. Carpio-Rivera E, Moncada-Jiménez J, Salazar-Rojas W, SoleraHerrera A. Acute effects of exercise on blood pressure: a meta-analytic investigation. Arq Bras Cardiol. 2016;106(5):422-433.

Diabetes, Metabolic Syndrome and Obesity: Targets and Therapy

Dovepress

\section{Publish your work in this journal}

Diabetes, Metabolic Syndrome and Obesity: Targets and Therapy is an international, peer-reviewed open-access journal committed to the rapid publication of the latest laboratory and clinical findings in the fields of diabetes, metabolic syndrome and obesity research. Original research, review, case reports, hypothesis formation, expert opinion and commentaries are all considered for publication. The manuscript management system is completely online and includes a very quick and fair peer-review system, which is all easy to use. Visit http://www.dovepress.com/testimonials.php to read real quotes from published authors. 